\title{
Practical design approach for trapezoidal modulation of a radio-frequency quadrupole
}

\author{
A. S. Plastun ${ }^{*}$ and P. N. Ostroumov \\ Facility for Rare Isotope Beams, Michigan State University, East Lansing, Michigan 48824, USA
}

(Received 18 December 2017; published 19 March 2018)

\begin{abstract}
Trapezoidal modulation of quadrupole electrodes offers additional benefits to the concept of a radiofrequency quadrupole (RFQ). Because of the significant increase of the effective shunt impedance, RFQs with trapezoidal modulation have a reduced interelectrode voltage or resonator length as compared to conventional RFQs with sinusoidal modulation. This feature is especially valuable for RFQs operating in cw mode, since it reduces the required rf power. We develop a detailed procedure for the design of RFQ electrodes with trapezoidal modulation. With our design procedure and by properly choosing the trapezoidal cell parameters, we can easily control the peak surface fields in the RFQ to the same level as for sinusoidal cell modulation. The procedure is applied to the design of the electrodes for the ReA3 RFQ at Michigan State University.
\end{abstract}

DOI: 10.1103/PhysRevAccelBeams.21.030102

\section{INTRODUCTION}

A radio-frequency quadrupole (RFQ) is the most essential section for radio-frequency (rf) ion accelerators nowadays. Both pulsed and continuous wave $(\mathrm{cw})$ mode ion accelerators have an RFQ section covering the energy range of ion beams from $(2-100) \mathrm{keV} / \mathrm{u}$ to about $(0.2-3) \mathrm{MeV} / \mathrm{u}$. The energy gain rate and hence the effective shunt impedance of RFQs decreases with the beam energy. However, most RFQ applications require high output beam energies and high shunt impedance [1-4]. For example, in superconducting (SRF) heavy-ion linacs, the RFQ should provide a high enough beam energy to enable the efficient use of available voltage from SRF cavities. High shunt impedance of the RFQ reduces the heat load on the resonator and helps to maintain stable and reliable operation in cw mode. Long RFQs with a reduced voltage can obviously provide the given beam energy at a reduced heat load. But resonators with the length of a few wavelengths are vulnerable to significant field distortion effects, such as field tilt along the resonator and the presence of dipole field components on the beam axis [5].

Applying trapezoidal modulation to the RFQ electrodes is an easy and efficient way to increase the transit-time factor of RFQ cells and, therefore, improve the energy gain and the effective shunt impedance of the RFQ. Cylindrical

\footnotetext{
Corresponding author. plastun@frib.msu.edu

Published by the American Physical Society under the terms of the Creative Commons Attribution 4.0 International license. Further distribution of this work must maintain attribution to the author(s) and the published article's title, journal citation, and DOI.
}

rods with trapezoidal modulation have been proposed for an RFQ for ease of electrode manufacturing [6,7]. The first RFQ was built and commissioned in the Institute for High Energy Physics (Protvino) in 1971 [8]. It had a steplike modulation of electrodes (see Fig. 1, top left), which was replaced by trapezoidal modulation with a simple piecewise linear profile soon (see Fig. 1, top right) [9]. Trapezoidal electrodes were easier to manufacture with a lathe machine than the electrodes with sinusoidal modulation. Such trapezoidal electrodes were widely used by the Institute for Applied Physics (Frankfurt) for four-rod RFQs [10]. However, the accurate calculation of the field distribution created by such electrodes required the numerical solution of the Laplace equation. Los Alamos National Laboratory proposed the wedge-shaped vane tips with a variable radius
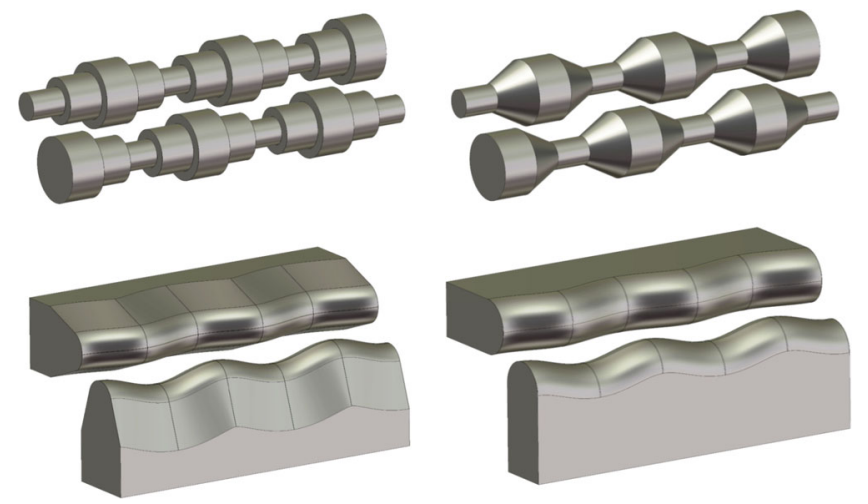

FIG. 1. RFQ rods and vane tips: top left, cylindrical rods with steplike modulation; top right, cylindrical rods with a linear slope of trapezoidal modulation; bottom left, wedge-shaped vane tips with sinusoidal modulation; bottom right, flat semicircular vane tips with sinusoidal modulation. 
of curvature along the electrode and sinusoidal modulation (see Fig. 1, bottom left) [11] to create a field distribution closely described by the so-called two-term potential function $[5,12]$. A three-dimensional (3D) numerically controlled milling machine with a ball-end tool was used to manufacture such vane tips. Flat semicircular vanes with a constant radius of the tip curvature and sinusoidal modulation (see Fig. 1, bottom right) were proposed at the Institute for Theoretical and Experimental Physics (Moscow) [13] to be machined by a two-dimensional (2D) milling machine equipped with a concave cutter tool. The wedge-shaped and flat semicircular vane tips both with sinusoidal modulation and a constant radius of the tip curvature are currently used in most RFQs. Trapezoidal modulation has been recalled only in 2000 with the purpose of improving the transit-time factor of RFQ cells [14]. In 2010, the smooth trapezoidal vane tip modulation was implemented in a cw RFQ [15] for the first time.

In 2017, the upgrade of the ReA3 RFQ [16] was initiated at the National Superconducting Cyclotron Laboratory (NSCL) of Michigan State University. The upgrade included the replacement of the modulated electrodes and stems of the four-rod $\mathrm{rf}$ structure. The new RFQ design reduces the operating interelectrode voltage by $19 \%$ in order to lower the rf heat load on the structure. Trapezoidal modulation of the electrodes has been applied in the regular part of the RFQ to keep the beam energy gain. Currently, no RFQ-design code capable of designing the electrodes with both sinusoidal and trapezoidal modulation is available. This paper presents a practical 3D approach, developed for the design of such electrodes. As a result of the new design procedure, we obtained the computer-aided design (CAD) model of the new electrodes for the ReA3 RFQ.

\section{DESIGN REQUIREMENTS}

The main purpose for the RFQ design is to develop the exact geometry of electrodes which satisfies the beam dynamics specifications. As a result, a CAD model of electrodes is created. We apply the following requirements for the CAD model of electrodes: (a) manufactured electrodes have exactly the same geometry as the CAD model; (b) manufactured electrodes provide the desired variation of the synchronous phase along the RFQ with a given accuracy; (c) the longitudinal profile of the electrodes is continuous and smooth; (d) the interelectrode voltage and midcell aperture radius of the RFQ can be variable along the RFQ electrodes; (e) the initial bunching section of the RFQ with a variable synchronous phase and modulation factor has sinusoidal electrode modulation; (f) the regular accelerating section of the RFQ has trapezoidal electrode modulation; (g) the transition from the initial section to the regular accelerating section of the RFQ has trapezoidal modulation with a variable modulation factor and synchronous phase; (h) the peak surface field in each RFQ cell is accurately calculated and distributed as uniformly as possible along the RFQ.
In order to satisfy requirement (a), we use flat semicircular electrodes (Fig. 1, bottom right), since they have a constant radius of tip curvature and can be machined with both standard concave and ball-end cutters. More details on the cutter path and cutter radius compensation are presented in the Appendix. For item (b), we use the accurately simulated fields of the CAD 3D model to design the RFQ electrodes' modulation. The electrodes are designed in the quasielectrostatic approximation. We believe that the rf effects on the voltage distribution are small and can be controlled during the $\mathrm{rf}$ design of the resonator if needed. The accurate electrostatic 3D simulations of the electrodes provide an easy way to control many parameters, such as the surface electric field distribution, the interelectrode capacitance distribution, etc. A combination of different software can be used to control the design process of the RFQ electrodes, build the CAD model, and simulate the electrostatic field as it was done in Refs. [17,18]. The VBA Macro Editor of CST STUDIO SUITE [19] has been proposed as a single tool to control, build, and simulate the accurate CAD models of RFQs [20]. This approach has been implemented in the design of the trapezoidal electrodes with a constant modulation factor and synchronous phase [21]. Our design approach is based on the CST macro, too, but we are not limited by the constant modulation factor and synchronous phase. Moreover, we use a continuous and smooth longitudinal profile of the electrode tip, which supports both sinusoidal and trapezoidal modulation of the RFQ cells.

\section{ELECTRODE PROFILE}

In order to make a continuous and smooth electrode profile along the whole RFQ, we developed a profile for a single RFQ cell, which provides positional and tangential continuity with the adjacent cells. Figure 2 shows the transverse and longitudinal profiles of the RFQ cell. The radius of the electrode tip curvature $R_{v}$ does not depend on the distance from the longitudinal axis to the electrode. The longitudinal profile of the $j$ th cell consists of two straight parts of zero slope tangents and a sinusoidal junction of the length $g_{j}$, which is called the accelerating gap of the trapezoidal RFQ cell. The total length of the $j$ th cell is $L_{j}$. Trapezoidal cells have $g_{j}<L_{j}$, while sinusoidal cells have $g_{j}=L_{j}$. Another two parameters define the $j$ th cell cross section at its entrance: $R_{j}$ is the average aperture radius at the $j$ th cell entrance, and $m_{j}$ is the modulation factor at the $j$ th cell entrance. The cross section at the end of the $j$ th cell is obviously the same as the entrance cross section of the $(j+1)$ th cell. The minimum aperture radius at the $j$ th cell entrance is defined as

$$
a_{j}(z)=\frac{2 R_{j}}{m_{j}+1} .
$$

The maximum aperture radius at the $j$ th cell entrance is equal to $m_{j} a_{j}$. The variation of the average aperture radius along the $j$ th cell with the center at $z=c_{j}$ follows the law 


$$
R_{0}(z)= \begin{cases}R_{j}, & z \in\left[c_{j}-\frac{L_{j}}{2}, c_{j}-\frac{g_{j}}{2}\right) ; \\ \frac{R_{j+1}+R_{j}}{2}+\frac{R_{j+1}-R_{j}}{2} \sin \left(\pi \frac{z-c_{j}}{g_{j}}\right), & z \in\left[c_{j}-\frac{g_{j}}{2}, c_{j}+\frac{g_{j}}{2}\right] \\ R_{j+1}, & z \in\left(c_{j}+\frac{g_{j}}{2}, c_{j}+\frac{L_{j}}{2}\right] .\end{cases}
$$

For example, if the average aperture radius remains constant along the RFQ, then $R_{0}(z)=R_{j}=R_{j+1}=R_{0}$. The common equations for the longitudinal profiles of horizontal $R_{x}(z)$ and vertical $R_{y}(z)$ electrodes are

$$
R_{x, y}(z)= \begin{cases}R_{0}(z)\left[1 \pm(-1)^{j} \frac{m(z)-1}{m(z)+1}\right], & z \in\left[c_{j}-\frac{L_{j}}{2}, c_{j}-\frac{g_{j}}{2}\right) ; \\ R_{0}(z)\left[1 \pm(-1)^{j+1} \frac{m(z)-1}{m(z)+1} \sin \left(\pi \frac{z-c_{j}}{g_{j}}\right)\right], & z \in\left[c_{j}-\frac{g_{j}}{2}, c_{j}+\frac{g_{j}}{2}\right] ; \\ R_{0}(z)\left[1 \pm(-1)^{j+1} \frac{m(z)-1}{m(z)+1}\right], & z \in\left(c_{j}+\frac{g_{j}}{2}, c_{j}+\frac{L_{j}}{2}\right],\end{cases}
$$

where $m(z)$ is a continuous and smooth function, which describes the variation of the modulation factor. The sign " \pm " should be replaced by "+" for the $x$-plane profile and by "-_" for the $y$-plane profile. The function $m(z)$ is given by

$$
m(z)= \begin{cases}m_{j}, & z \in\left[c_{j}-\frac{L_{j}}{2}, c_{j}-\frac{g_{j}}{2}\right) \\ \frac{m_{j+1}+m_{j}}{2}+\frac{m_{j+1}-m_{j}}{2} \sin \left(\pi \frac{z-c_{j}}{g_{j}}\right), & z \in\left[c_{j}-\frac{g_{j}}{2}, c_{j}+\frac{g_{j}}{2}\right] \\ m_{j+1}, & z \in\left(c_{j}+\frac{g_{j}}{2}, c_{j}+\frac{L_{j}}{2}\right] .\end{cases}
$$

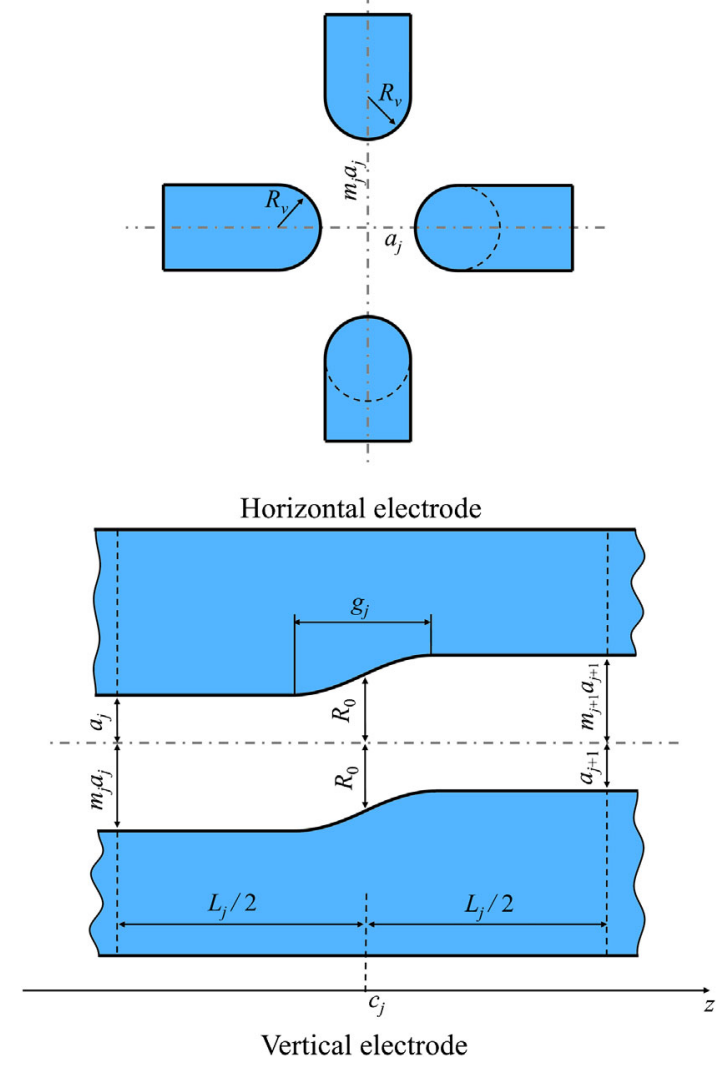

FIG. 2. Transverse (top) and longitudinal (bottom) profiles of an RFQ cell.
The sinusoidal law for the modulation factor is used within the accelerating gap. However, modulation can follow any other smooth function, which is symmetrical about the center of the cell and which has a zero slope at the gap ends, for example, a polynomial function given by

$$
\begin{aligned}
m(z)= & \frac{m_{j+1}+m_{j}}{2}+\frac{m_{j+1}-m_{j}}{2} \\
& \times\left[3\left(\frac{z-c_{j}}{g_{j}}\right)-4\left(\frac{z-c_{j}}{g_{j}}\right)^{3}\right], \\
z \in & {\left[c_{j}-\frac{g_{j}}{2}, c_{j}+\frac{g_{j}}{2}\right] }
\end{aligned}
$$

or multiharmonic function given by

$$
\begin{aligned}
m(z)= & \frac{m_{j+1}+m_{j}}{2}+\frac{m_{j+1}-m_{j}}{2}\left[(1-p) \sin \left(\pi \frac{z-c_{j}}{g_{j}}\right)\right. \\
& \left.-p \sin \left(3 \pi \frac{z-c_{j}}{g_{j}}\right)\right], \\
z \in & {\left[c_{j}-\frac{g_{j}}{2}, c_{j}+\frac{g_{j}}{2}\right] }
\end{aligned}
$$

where $p$ is the relative amplitude of the third harmonic of the function. Figure 3 shows plots for different modulation functions and electrode profiles for a sinusoidal half-cell. The polynomial function (5) is very close to the sinusoidal 

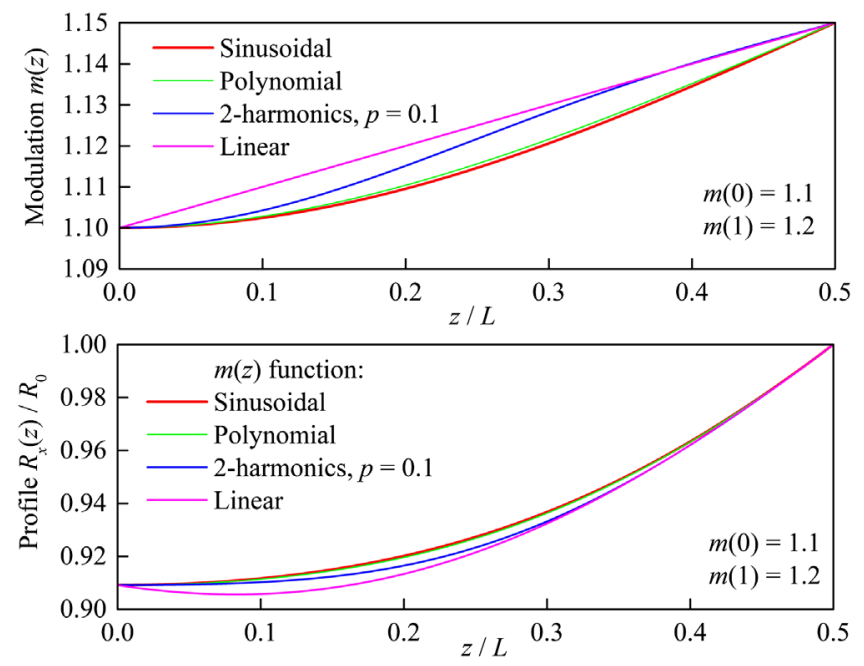

FIG. 3. Modulation law functions and electrode profiles within the accelerating gap.

function of Eq. (4); therefore, the profiles are close, too. The parameter $p$ of the function (6) provides control of the longitudinal profile curvature, which can be used, for example, to reduce the peak fields on the electrode's surface. The linear function for the modulation factor is simple but will lead to an offset of the minima and maxima of the longitudinal profile as shown in Fig. 3 in the magenta curve. The minimum of the profile is located at $z / L \approx 0.08$. The magnitude of the offset increases with the modulation growth rate $\left(m_{j+1}-m_{j}\right)$. Such offsets are not acceptable, since they change the cells' lengths and even shift cells' centers along the $z$ axis. The advances of the synchronous phase due to the shifts are difficult to control, which contradicts item (b) in the list of the requirements. Similar shifts of the longitudinal profile happen with any other function $m(z)$ with a nonzero slope at the ends of the accelerating gap. Moreover, the electrode profile built with a nonsmooth function $m(z)$ is not smooth, too.

It is worth mentioning that profiles (3) with the modulation function (4) are limited by the modulation growth rate. If the modulation factor changes very fast from cell to

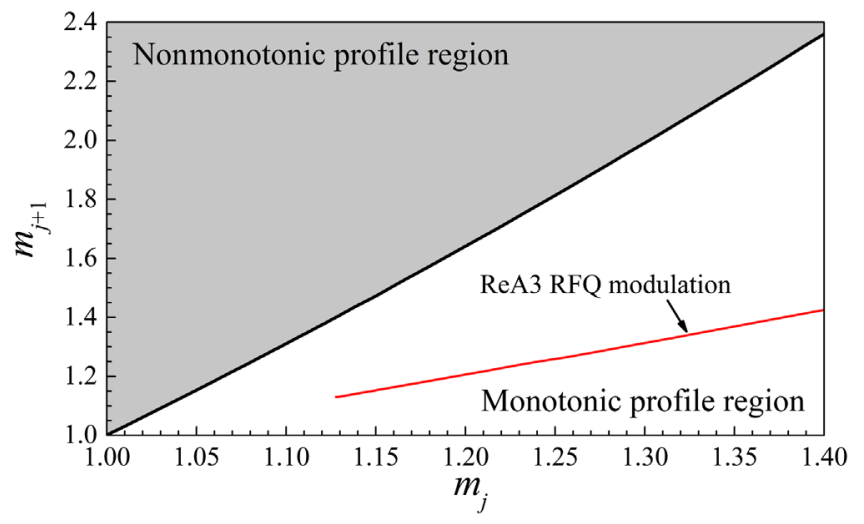

FIG. 4. Limitation of the modulation growth rate. cell, the electrode profile may become nonmonotonic within the cell, because function (4) changes faster than (3). Fortunately, the modulation growth rate $\left(m_{j+1}-m_{j}\right)$ of practical designs is usually below the limit shown in Fig. 4. The limiting curve was numerically calculated for the case of $R_{0}(z)=R_{0}$. It does not depend on the accelerating gap length, since both the modulation and profile functions have the same arguments of the sine function.

\section{ACCELERATING GAP}

The optimal design of a trapezoidal cell provides the same peak surface electric field both inside and outside the region of the accelerating gap. Figure 5 shows the field enhancement factor $\kappa=E_{\text {peak }} R_{0} / U$ obtained with an electrostatic simulation of the cell with a length of $10 R_{0}$ and a radius of the tip curvature of $0.8 R_{0}$, where $E_{\text {peak }}$ is the peak electric field on the electrodes' surface and $U$ is the voltage applied to the RFQ electrodes. All results presented below correspond to flat-semicircular electrodes with a radius of vane tip curvature of $0.8 R_{0}$. The optimal length of the accelerating gap is about $2.8 R_{0}$. It provides the maximum $T / \kappa$ ratio, i.e., the highest energy gain rate at a given magnitude of the peak surface field and average aperture radius. If the gap length is smaller than $2.8 R_{0}$, then the peak surface field does not depend on the length of the trapezoidal cell, because it is reached in the accelerating gap region, which remains the same at any cell length. It can be beneficial for the RFQ design to make peak fields independent from the cell length. Figure 6 shows the field enhancement factor for a cell with $g=2.6 R_{0}$. It remains constant until the cell is short and close to the sinusoidal one at $g / L>0.7$. In this case, peak fields are driven mostly by the accelerating gap, not by the transverse profile of electrodes. If one assumes constant $R_{0}$, then $g$ remains

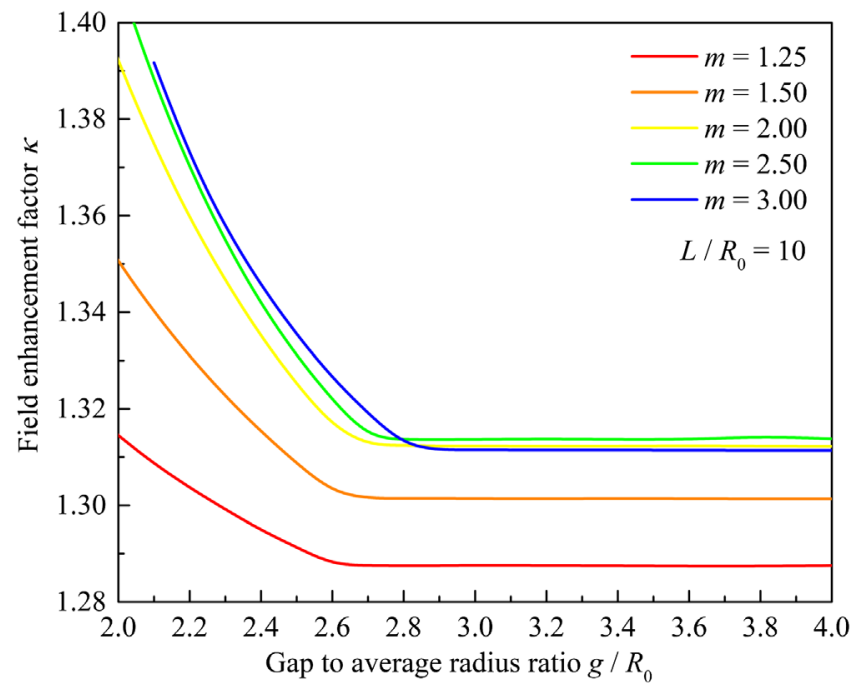

FIG. 5. Field enhancement factor of the RFQ cell with trapezoidal modulation. 


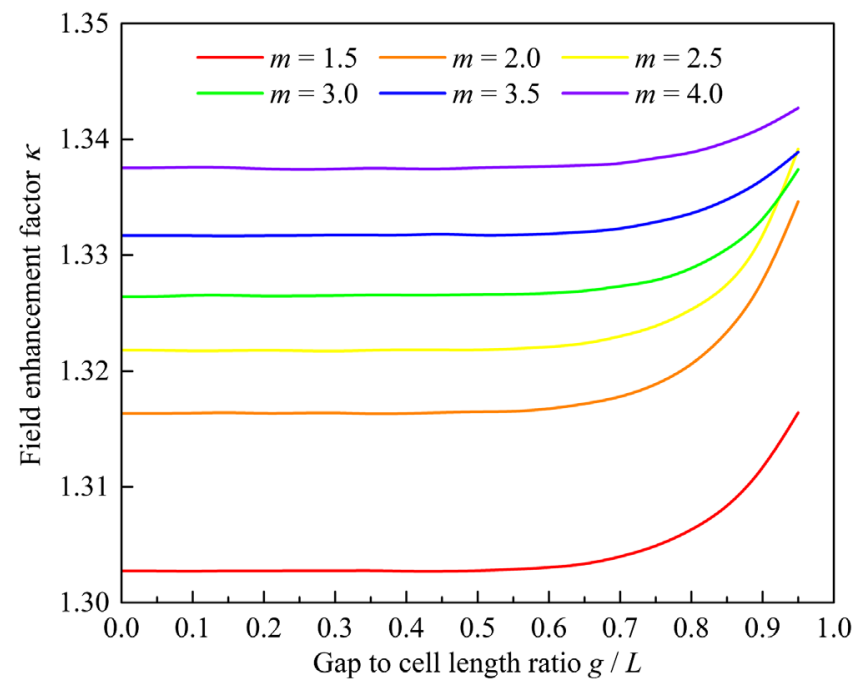

FIG. 6. Field enhancement factor of the RFQ cell with trapezoidal modulation at $g / R_{0}=2.6$.

constant, too, while the $L$ is variable, because the horizontal axis is $g / L$. It means that the left part of the plot $(g / L<0.7)$ corresponds to long trapezoidal cells, while the right part of the plot $(g / L>0.7)$ to short sinusoidal cells with the same sinusoidal gap length. The field enhancement factor increases for short cells, because peaks fields are not driven only by the transverse profile of the electrode. A small curvature radius of the longitudinal profile may dominate for short cells. Therefore, Fig. 6 means that the peak fields of a short sinusoidal cell could be larger than that of the long cell with trapezoidal modulation.

The variation of the transit-time factor is presented in Fig. 7. Curves in the figure tend well to the theoretical limits: $T=1.0$ at $g / L=0$ and $T=\pi / 4 \approx 0.785$ at $g / L=1$. However, we have not found a simple and precise

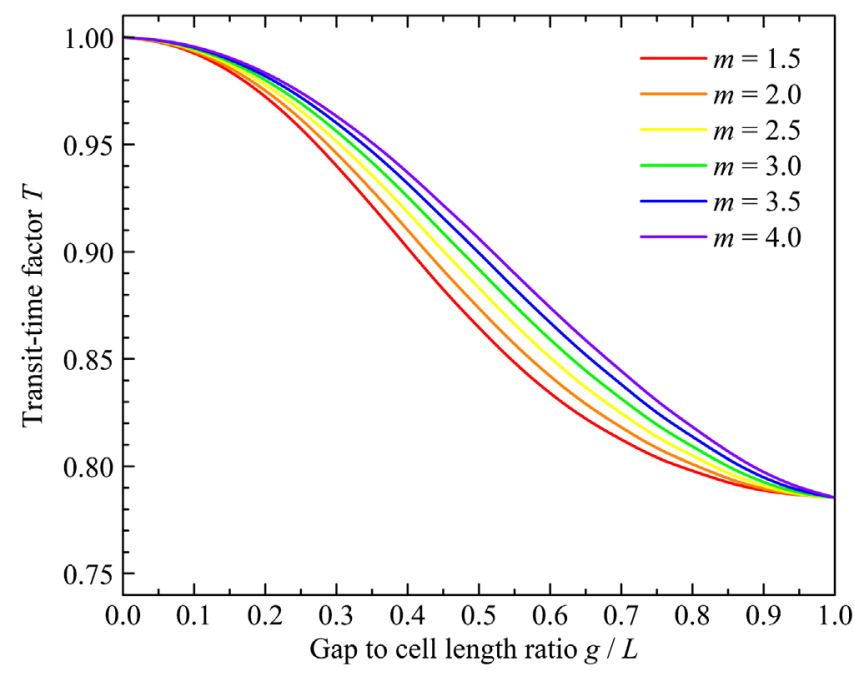

FIG. 7. Transit-time factor of the RFQ cell with trapezoidal modulation at $g / R_{0}=2.6$. analytical formula for the transit-time factor in the whole range of $g / L$.

\section{3D MODEL}

The goal of the design procedure is to calculate the parameters for each RFQ cell: average radius of aperture, modulation factor, gap length, and cell length. The modulation design includes two steps. The first step is an estimate of the output energy, total length, and cells' parameters. A primary 3D CAD model of the RFQ electrodes is created as a result of this step. This model is created in CST for the following electrostatic simulations. At the second step, the primary 3D model is modified cell by cell to provide the desired law of synchronous phase, modulation, and average radius of aperture along the electrodes with a given accuracy. For the ReA3 RFQ, we assumed a constant average aperture radius of $6.56 \mathrm{~mm}$. The length of the accelerating gap of all trapezoidal cells is equal to $15 \mathrm{~mm}$.

The primary 3D model is designed using a simple and fast Fortran code. It usually takes several iterations to obtain the desired combination of the output energy, RFQ length, acceptable initial synchronous phase, and modulation factor. A flowchart of the design algorithm to generate the primary 3D model for ReA3 RFQ is shown in Fig. 8. A short description is given below. (i) Define the initial values of synchronous phase $\varphi$, acceleration efficiency $A$, and transit-time factor $T$ [5] at the input normalized velocity of the reference particle $\beta=v / c$. (ii) Start from the first cell. (iii) Evaluate the synchronous phase $\varphi$ and the product $A T$ of the acceleration efficiency $A$ and the transit-time $T$ factor with the equations for adiabatic bunching in the RFQ:

$$
\begin{aligned}
\left(\frac{\varphi_{0}}{\varphi}\right)^{2} & =\frac{W}{W_{0}}, \\
\frac{A T}{A_{0} T_{0}} & =\frac{W \sin \varphi_{0}}{W_{0} \sin \varphi},
\end{aligned}
$$

where $W$ is the beam kinetic energy. Index " 0 " means the initial values for the RFQ or the values for the first RFQ cell. Nonindexed parameters are the values in an arbitrary cell in the RFQ. (iv) Calculate the length of the cell using the average value of $\beta$ along the cell. (v) If the cell is longer than $15 \mathrm{~mm}$, the cell is trapezoidal with a 15-mm-long accelerating gap. (vi) Search for the modulation factor $m$, which provides $A T$ evaluated from the equations. (vii) If the calculated $m>2.5$, then apply the limit $m=2.5$ and recalculate the length of the cell. (viii) Repeat for the following cells.

The Laplace equation is solved numerically at each cell to find the modulation factor. If the current value of $m$ does not provide the required $A T$, then $m$ is changed according to the binary search (bisection) algorithm and the Laplace equation is solved again. This process repeats until the given accuracy for $m$ is achieved. The solver for the Laplace equation uses a 


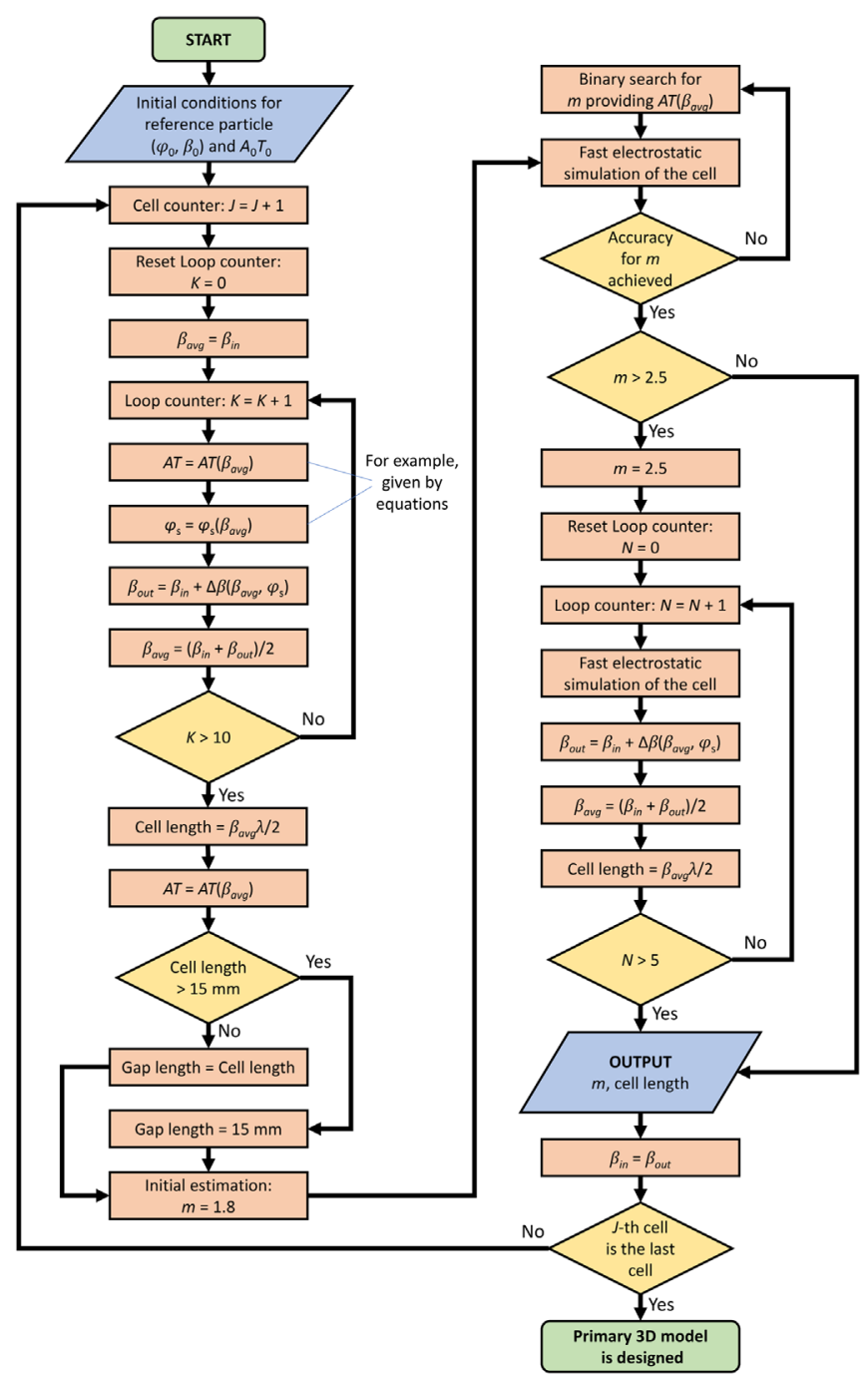

FIG. 8. Flowchart of the design algorithm of the primary 3D model.

finite-difference method and a mesh with $96 \times 96 \times 40$ points in a box with dimensions $(6 a) \times(6 a) \times L$, where $a$ is the minimum aperture radius of the cell. Each run of the whole algorithm to design 100 cells takes about $30 \mathrm{~min}$. This time can be significantly reduced, if one uses the analytical formulas for acceleration efficiency $[5,12]$ of sinusoidal cells instead of a numerical solution of the Laplace equation to search for the modulation factor. In this case, some adjustments of the modulation factor can be performed in CST during the second step of the electrodes' design.

Figure 9 shows an example of the full modeling procedure of a single RFQ cell. The full-length electrode may consist of several hundreds of cells. Our first CST VBA macro was developed to create the curve object for the whole electrode profile of the primary 3D model using Eqs. (1)-(4). The curve and the lines limiting the electrode boundaries form the closed planar circuit shown in Fig. 10, which is extruded into a solid. Some edges of the solid are rounded. Figure 11 presents a 3D CAD model of two

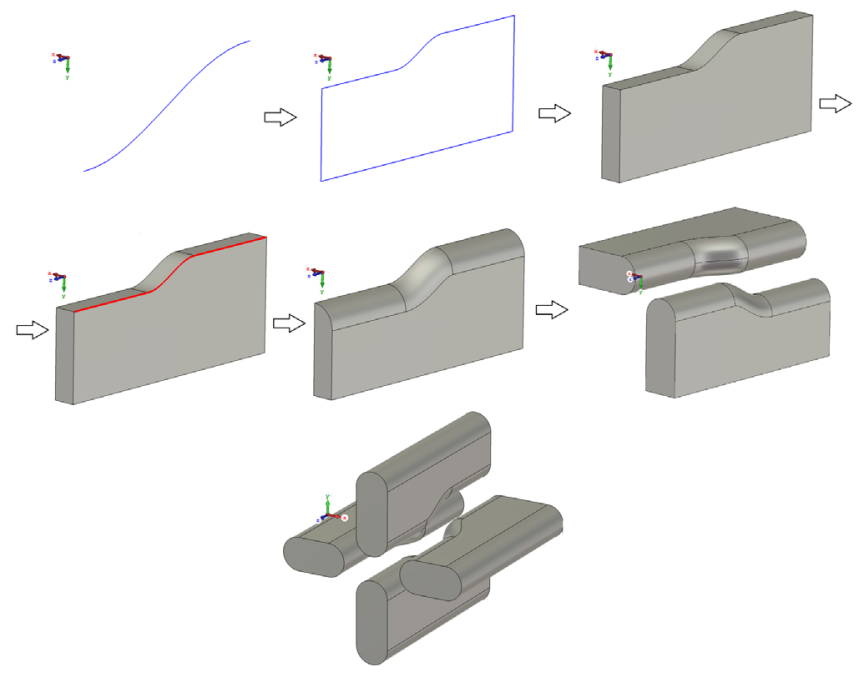

FIG. 9. Modeling steps of a 3D model for the RFQ cell.

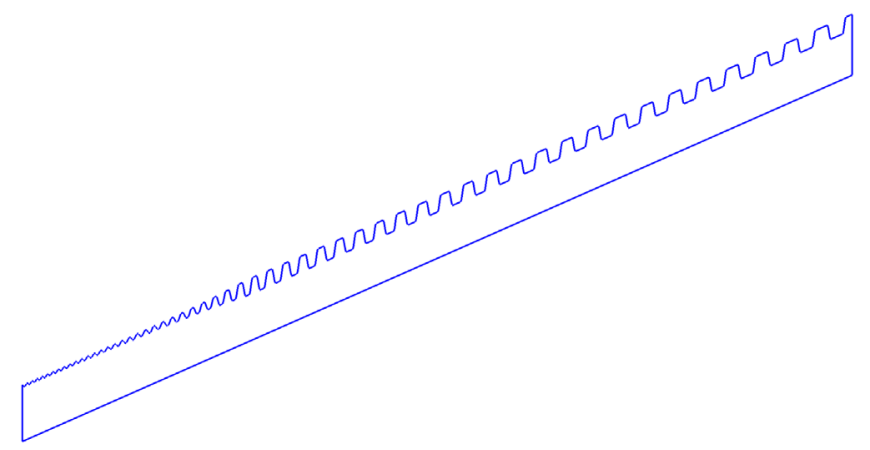

FIG. 10. Closed contour for a 3D model of the RFQ electrode.

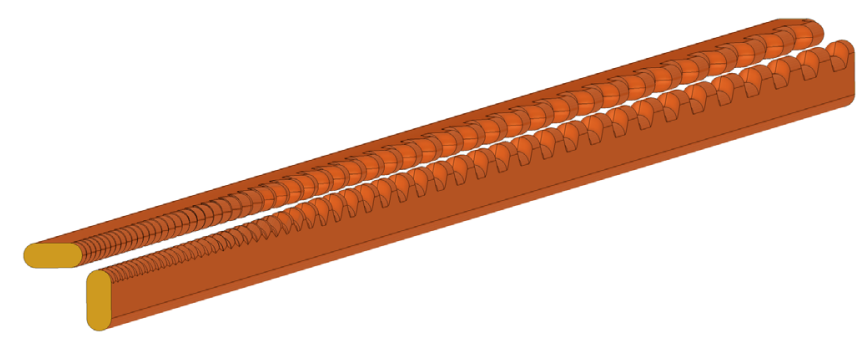

FIG. 11. A 3D model of the ReA3 RFQ electrodes.

adjacent electrodes of the ReA3 RFQ. We define the geometry of each cell by variables stored in the parameter list of CST. The macro uses the names of these variables to create the profile curve. This approach provides the instant modification of the geometry with no need to execute the macro every time we change any geometrical parameter.

We developed a second CST macro code to perform the accurate adjustments of the RFQ cells automatically. This macro changes the values of the variables in the parameter list of the primary 3D model. Flowchart of this macro is presented in FIG. 12. The main steps of the macro are: (i) Initial value of the synchronous phase $\varphi_{0}$ is assumed at 


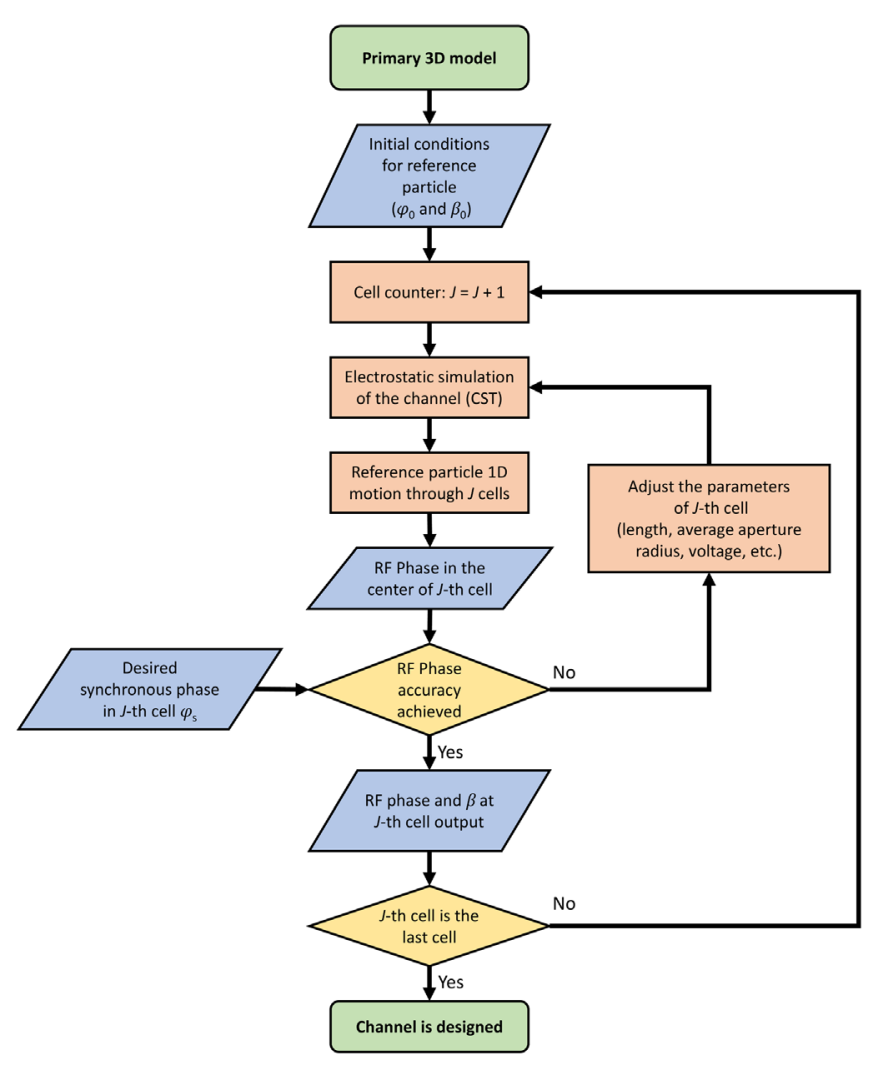

FIG. 12. Flowchart of the design algorithm of RFQ electrodes' modulation.

initial $\beta_{0}$ in the center of the 1 st cell. (ii) Start from the second cell. (iii) Perform the electrostatic simulation of the primary 3D model in CST. (iv) Perform a 1D simulation of the reference particle longitudinal dynamics. (v) Evaluate the rf phase $\varphi=\omega t$ when the particle is in the geometrical center of the cell, where $\omega$ is the circular frequency of the $\mathrm{rf}$ field and $t$ the time. We assume that the electrical center of the cell coincides with its geometrical center; i.e., this rf phase is the synchronous phase. (vi) If the rf phase is different from the required value, then adjust the length of the cell properly. This step may also include adjustments of the modulation factor (i.e., acceleration efficiency) to follow the adiabatic bunching concept more accurately than provided by the primary $3 \mathrm{D}$ model. If the average aperture radius is variable along the RFQ, it can be corrected at this step, too. (vii) Repeat the electrostatic and reference particle dynamics simulations until the given accuracy for the $\mathrm{rf}$ phase of the reference particle in the cell center is achieved. (viii) Repeat for the following cells.

We prefer to simulate the full-length RFQ electrodes at each iteration of cell length adjustments rather than single cells. It may help to avoid possible errors caused by artificial longitudinal boundary conditions. Each run of the CST electrostatic simulation of the full-length RFQ electrodes takes about 10-30 min. Figure 13 shows the tetrahedral mesh of the CST model.

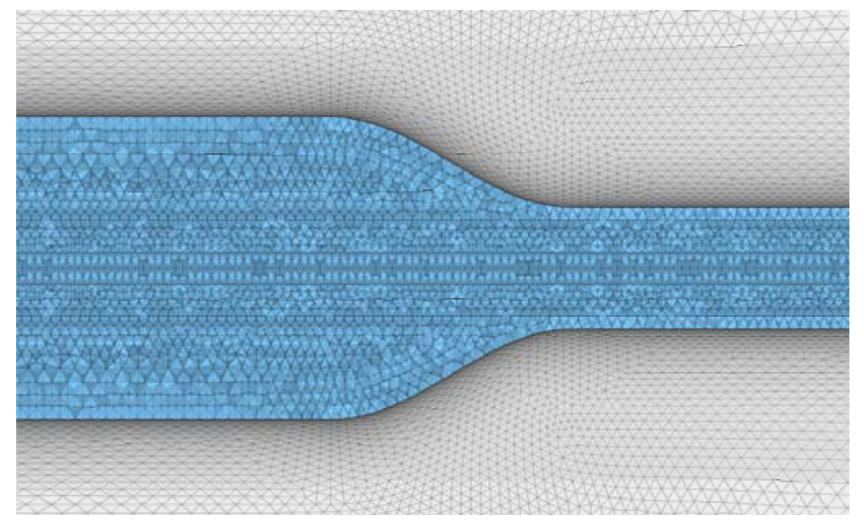

FIG. 13. Tetrahedral mesh of the full-length 3D model of the RFQ electrodes in a longitudinal cross section.

The simulation of the longitudinal dynamics of the reference particle starts from the beginning of the RFQ at every iteration of length adjustments. This is done to avoid unexpected advances of the rf phase, induced in previous cells by the length adjustment process. They may be as high as the desired accuracy for the rf phase-a fraction of a degree.

The automatization of electrodes' geometry adjustments by a CST macro significantly reduces the inconvenience from the long simulation runtime. The total time of the fully automatic adjustment process of the ReA3 RFQ electrodes was about $40 \mathrm{~h}$ per 100 cells. Obviously, this time can be reduced to several hours if one simulates short parts of the electrodes and takes care of accurate and correct reference particle dynamics along these parts.

\section{DESIGN ANALYSIS}

The peak surface electric field and rf defocusing strength are the key parameters to be controlled during the design of the RFQ with trapezoidal modulation. For the highest accuracy, we calculate the peak surface electric field magnitude for each RFQ cell using the model of the single RFQ cell (see Fig. 9). Using the macro, we consequently apply the dimensions of each RFQ cell to the single-cell model, run the electrostatic simulation, and save the magnitude of the peak surface field. Figure 14 shows the distribution of the peak field along the ReA3 RFQ calculated with the macro. One can see the constant peak field magnitude in the section with trapezoidal modulation $(z>1 \mathrm{~m})$, since we keep the length of the accelerating gap constant and shorter than $2.8 R_{0}$. In other words, peak fields of these cells are driven by an accelerating gap. A similar macro is used to study the surface field effects from the random misalignments of the electrodes.

In order to estimate the rf defocusing effect, we calculate the transverse phase advance $\sigma$ per focusing period [5] for the particles at all $\mathrm{rf}$ phases in the range $\left(-180^{\circ}, 180^{\circ}\right)$. The transverse phase advance $\sigma$ for any particle of the beam can be derived from the elements of the matrix of the focusing period $M$ : 


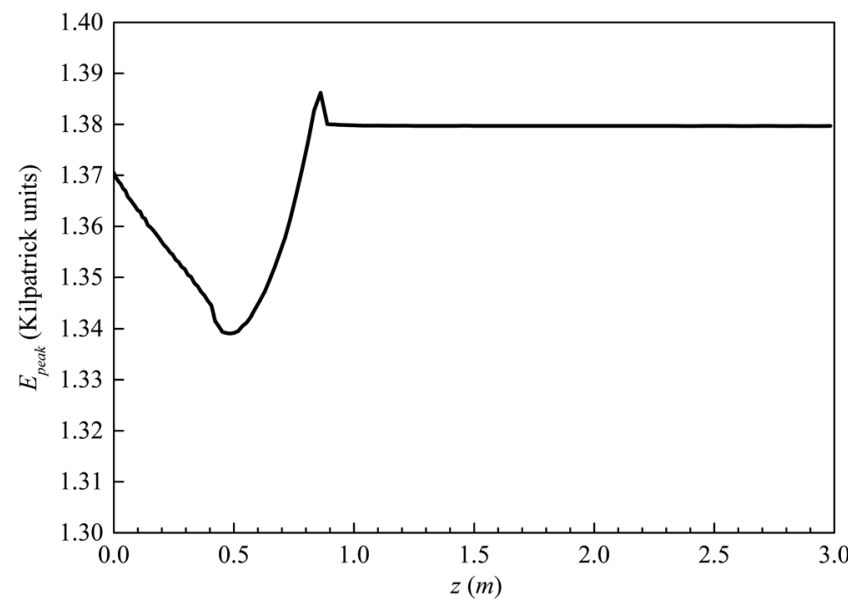

FIG. 14. Peak surface electric field along the ReA3 RFQ.

$$
\cos \sigma=\frac{M_{11}+M_{22}}{2} .
$$

Matrix $M$ for the $x$ plane is given by

$$
\left(\begin{array}{l}
x_{1} \\
x_{1}^{\prime}
\end{array}\right)=\left(\begin{array}{ll}
M_{11} & M_{12} \\
M_{21} & M_{22}
\end{array}\right)\left(\begin{array}{c}
x_{0} \\
x_{0}^{\prime}
\end{array}\right),
$$

where index " 0 " means coordinate and divergence of a particle at the entrance of the focusing period and index "1" means coordinate and divergence of a particle at the exit of the focusing period. Assuming $x_{0}^{\prime}=0$, we can get the element $M_{11}=x_{1} / x_{0}$. Assuming $x_{0}=0$, we get the element $M_{22}=x_{1}^{\prime} / x_{0}^{\prime}$. In both cases, the coordinate and divergence of a particle are taken from the result of the particle dynamics simulation through the focusing period. Similar calculations can be applied to the $y$ plane. Figure 15

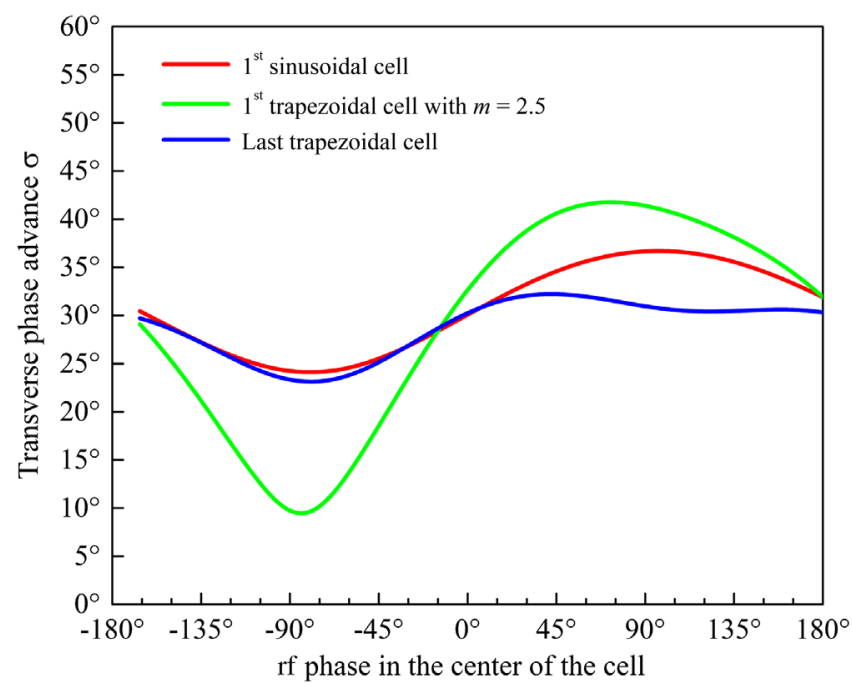

FIG. 15. Transverse phase advance at three different locations along the ReA3 RFQ. presents the transverse phase advance plot at different locations along the ReA3 RFQ. The strongest rf defocusing is achieved at the first trapezoidal cell with $m=2.5$, because this cell has the largest ratio of accelerating field magnitude to the beam kinetic energy. Stability of the transverse motion of the beam particles is maintained while $\sigma>0$.

\section{DISCUSSIONS}

The proposed design procedure has some limitations. For example, it assumes that laws for acceleration efficiency $A(W)$ and synchronous phase $\varphi(W)$ are known in advance. These parameters cannot be modified consistently with the longitudinal phase-space plot of the whole beam, because only the reference particle simulation is performed. Space-charge effects are ignored for the same reason. Also, we assume that the synchronous phase is equal to the phase of the rf field when the reference particle is at the geometrical center of the cell [12]. The value of this $\mathrm{rf}$ phase is easily derived from the simulation results.

\section{CONCLUSION}

Beam dynamics has been simulated with the TRACK code [22], using a 3D field of CST model. The simulation shows that the RFQ design completely satisfies both the beam dynamics and geometrical requirements. No transverse emittance growth was observed. Detailed simulation results on the ReA3 RFQ upgrade, which also include the redesign of the rf structure and its cooling circuits, will be published in a future paper.

We believe the presented approach and algorithms for the practical design of the RFQ electrodes will motivate designers to apply trapezoidal modulation. The theoretical limit for the gain of the shunt impedance provided by the trapezoidal modulation is about $(4 / \pi)^{2}-1 \approx 62 \%$. This value comes from the ratio of maximum transit-time factors for trapezoidal and sinusoidal cells. Practical values of the gain, averaged over the RFQ length, usually do not exceed $40 \%$. Our studies show that the properly designed trapezoidal electrodes do not compromise with their focusing capability and peak surface fields as compared to sinusoidal modulation.

\section{ACKNOWLEDGMENTS}

The authors thank Dr. Q. Zhao for comments on ReA3 RFQ design and Dr. A. C. C. Villari for continuing support. This material is based upon work supported by the National Science Foundation under Grant No. PHY-1565546.

\section{APPENDIX: CUTTER RADIUS COMPENSATION}

Milling of the modulation on the RFQ electrodes is an important process for the performance of the RFQ. 


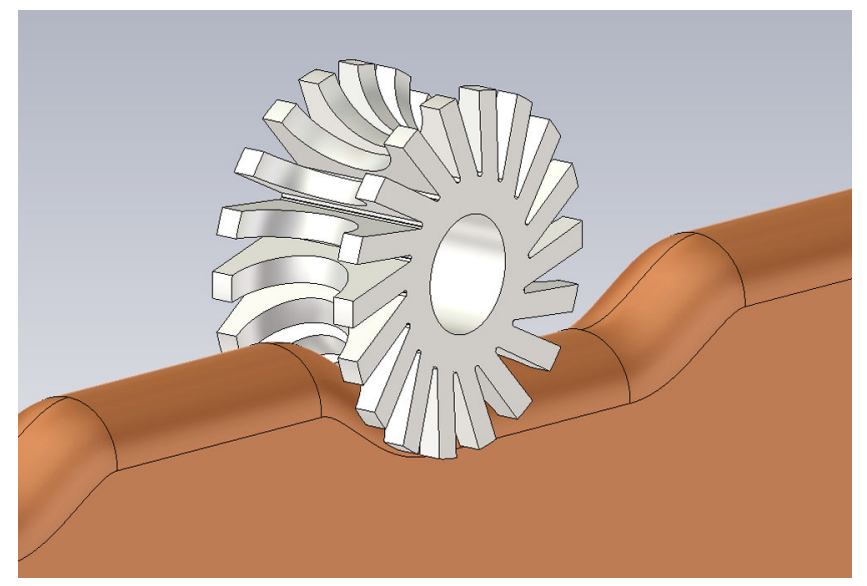

FIG. 16. A 3D model of the concave cutter and RFQ electrode.

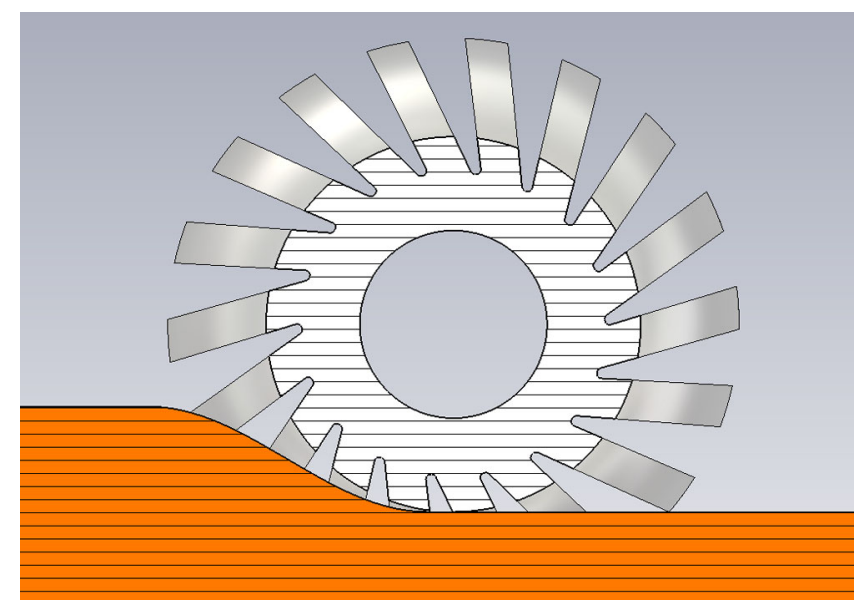

FIG. 17. The side view of the concave cutter and RFQ electrode.

Computer numerical control (CNC) milling machines can either calculate the cutter-tool path from the 3D model of the electrode by themselves, or RFQ designers provide the data for the cutter. Usually one of two types of cutter are used to machine the modulation - a concave cutter, schematically shown in Figs. 16 and 17, or a ball-end cutter, a simplified model of which is presented in Figs. 18 and 19. During the milling process, the cutter should follow the cutter path, which is defined for the geometrical center of the cutter. The cutter path is usually defined as a $2 \mathrm{D}$ or 3D curve.

A concave cutter requires only a $2 \mathrm{D}$ milling machine and 2D path. The 3D path for a ball-end cutter is much more complicated and requires a 5D-milling machine, because the cutter axis is usually kept normal to the milling surface as shown in Fig. 19. Below, we consider the 2D path for the concave cutter. However, the following results are correct for the ball-end cutter as well.

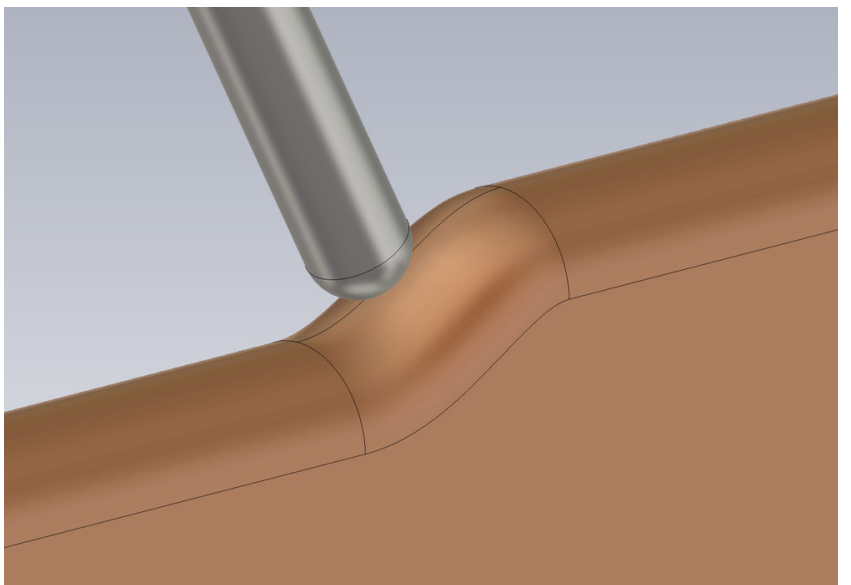

FIG. 18. A 3D model of the ball-end cutter and RFQ electrode.

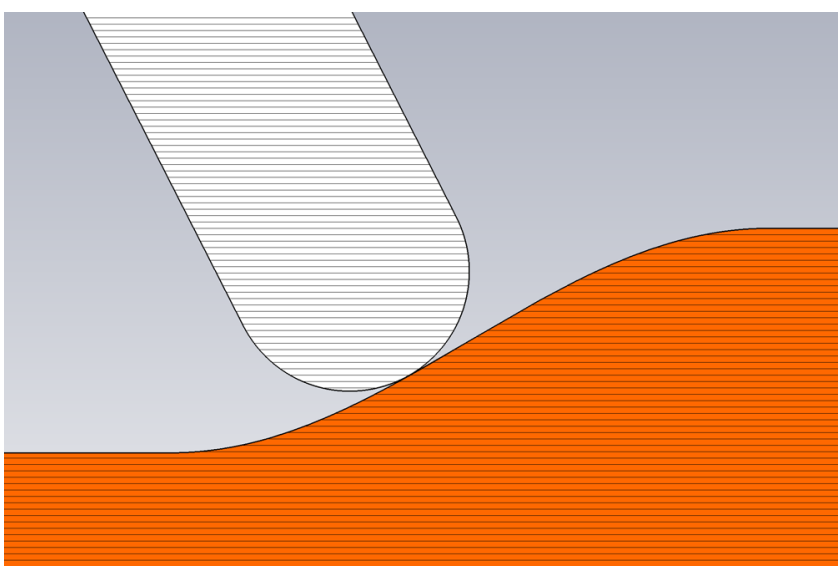

FIG. 19. The side view of the ball-end cutter and RFQ electrode.

It may seem that the cutter path follows the RFQ electrode profile $y(z)$ translated vertically by a cutter radius $R_{c}$ as shown in Fig. 20 by a red curve. But it is not true for any $R_{c}>0$. Equations (10)-(15) describe the cutter's center path with cutter radius compensation (blue curve in Fig. 20). Here $\alpha$ is the tangential angle of the profile, $\beta$ is the angle of the profile normal, $\Delta y(z)=y_{t}-y_{c}$, and $\Delta z(z)=z_{t}-z_{c}$ is the offset of the cutter center from the point of tangency of the cutter outline and the electrode tip (see Fig. 20).

$$
\begin{gathered}
\alpha(z)=\arctan y^{\prime}(z), \\
\beta(z)=\alpha(z)-\pi / 2, \\
\Delta y(z)=\frac{-R_{c}|\tan \beta(z)|}{\sqrt{1+|\tan \beta(z)|^{2}},}
\end{gathered}
$$



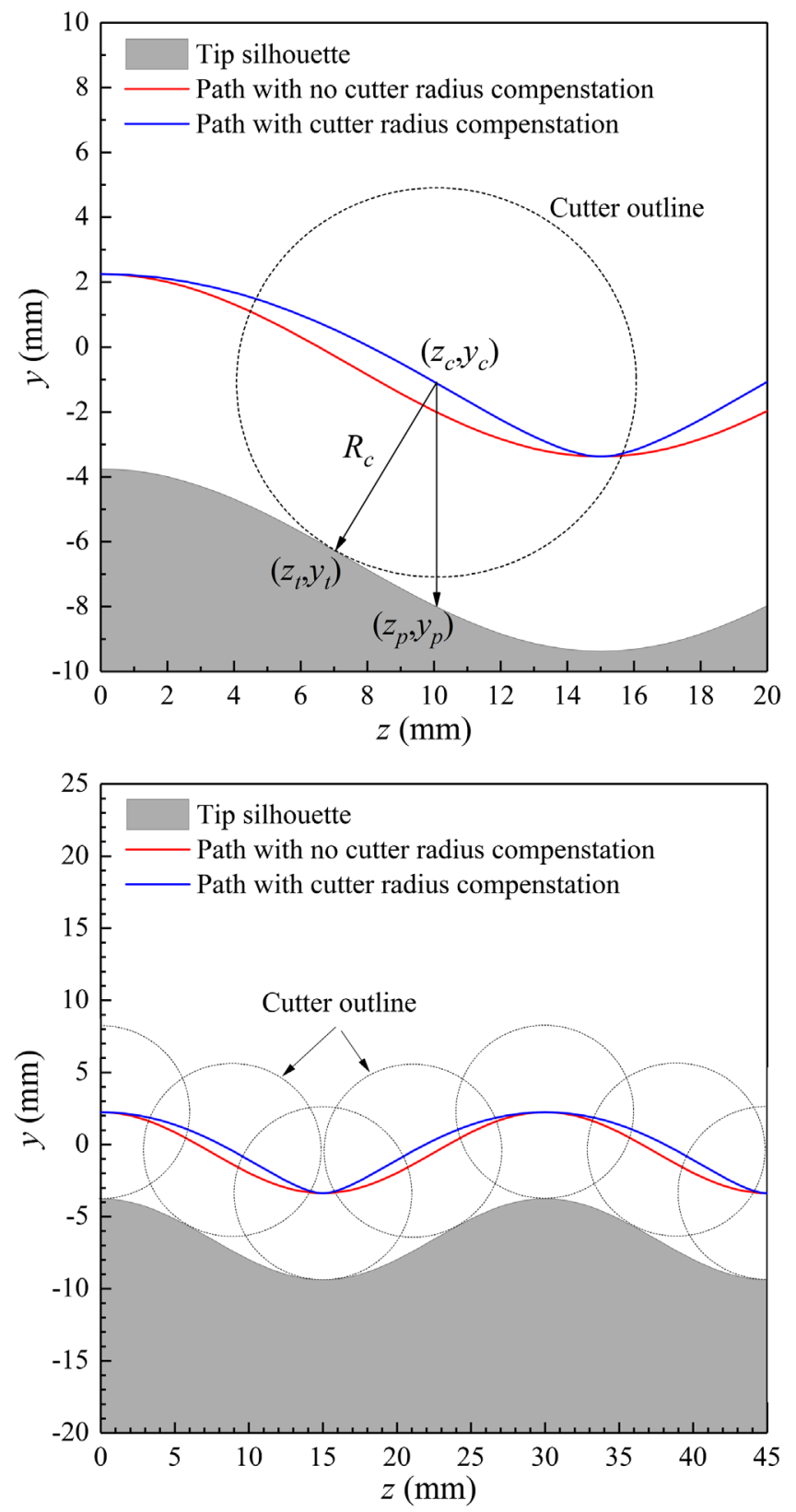

FIG. 20. The cutter-tool path and RFQ profile.

$$
\begin{gathered}
\Delta z(z)=\frac{\Delta y(z)}{\tan \beta(z)}, \\
z_{c}(z)=z+\Delta z(z), \\
y_{c}(z)=y(z)+\Delta y(z) .
\end{gathered}
$$

[1] P. N. Ostroumov, B. Mustapha, A. Barcikowski, C. Dickerson, A. A. Kolomiets, S. A. Kondrashev, Y. Luo, D. Paskvan, A. Perry, D. Schrage, S. I. Sharamentov,
R. Sommer, W. Toter, and G. Zinkann, Development and beam test of a continuous wave radio frequency quadrupole accelerator, Phys. Rev. Accel. Beams 15, 110101 (2012).

[2] M. Comunian, A. Pisent, E. Fagotti, and P. A. Posocco, Beam dynamics of the IFMIF-EVEDA RFQ, in Proceedings of the 11th European Particle Accelerator Conference, Genoa, 2008 (EPS-AG, Genoa, Italy, 2008), p. 3536.

[3] M. Vretenar, A. Dallocchio, V. A. Dimov, M. Garlasché, A. Grudiev, A. M. Lombardi, S. Mathot, E. Montesinos, and M. Timmins, A compact high-frequency RFQ for medical applications, in Proceedings of the 2014 Linear Accelerator Conference, Geneva, Switzerland (JACoW, Geneva, 2014), p. 935.

[4] A. S. Plastun, B. Mustapha, A. Nassiri, P. N. Ostroumov, L. Faillace, S. V. Kutsaev, and E. A. Savin, Beam dynamics studies for a compact carbon ion linac for therapy, in Proceedings of the 2016 Linear Accelerator Conference, East Lansing, MI (JACoW, East Lansing, 2017), p. 946.

[5] T. P. Wangler, $R F$ Linear Accelerators (Wiley-VCH, Weinheim, 2008).

[6] I. M. Kapchinskiy and V. A. Teplyakov, A linear ion accelerator with spatially uniform hard focusing, Prib. Tekh. Eksp. 2, 19 (1970).

[7] I. M. Kapchinskiy and N. V. Lazarev, The linear accelerator structures with space-uniform quadrupole focusing, in Proceedings of the 1979 Particle Accelerator Conference, San Francisco, CA (IEEE, New York, 1979), p. 3462.

[8] A. P. Maltsev, V. B. Stepanov, and V. A. Teplyakov, Institute for High Energy Physics, Serpukhov Report No. 71-116, 1971 (in Russian).

[9] B. M. Gorshkov et al., Institute for High Energy Physics, Serpukhov Report No. 76-139, 1976 (in Russian).

[10] A. Schempp, P. Junior, H. Klein, M. Daehne, M. Ferch, K. Langbein, and N. Zoubek, Status of the Frankfurt zeromode proton RFQ, in Proceedings of the 1983 Particle Accelerator Conference, Santa Fe, NM (IEEE, New York, 1983), p. 3536.

[11] C. W. Fuller, S. W. Williams, and J. M. Potter, Mechanical design considerations in FMIT RFQ development, in Proceedings of the 1979 Linear Accelerator Conference, Montauk, NY, edited by R. L. Witkover (Brookhaven National Laboratory, Upton, NY, 1979), p. 401.

[12] I. M. Kapchinskiy, Theory of Resonance Linear Accelerators (Harwood, Chur, Switzerland, 1985).

[13] R. M. Vengrov, E. N. Daniltsev, I. M. Kapchinskij, A. M. Kozodaev, V. V. Kushin, N. V. Lazarev, A. A. Nikitin, and I. V. Chuvilo, The pulsed proton prototype of a high current ion linac, in Proceedings of the 1981 Linear Accelerator Conference, Santa Fe, NM, edited by R. A. Jameson and Louise S. Taylor (Los Alamos National Laboratory, Los Alamos, NM, 1981), p. 92.

[14] O. K. Belyaev, O. V. Ershov, I. G. Maltsev, V. B. Stepanov, S. A. Strekalovskikh, V. A. Teplyakov, and A. V.Zherebtsov, IHEP experience on creation and operation of RFQs, in Proceedings of the 20th International Linac Conference, LINAC-2000, Monterey, CA, 2000 edited by A. W. Chao (SLAC, Menlo Park, CA, 2000), p. 259. 
[15] B. Mustapha, A. A. Kolomiets, and P. N. Ostroumov, Full 3d modeling of a radio-frequency quadrupole, in Proceedings of the 25th International Linear Accelerator Conference, LINAC-2010, Tsukuba, Japan (KEK, Tsukuba, Japan, 2010), p. 542.

[16] D. Leitner et al., Commissioning results of the REA RFQ at MSU, in Proceedings of the 24th Particle Accelerator Conference, PAC-2011, New York, 2011 (IEEE, New York, 2011), p. 1912.

[17] S. Jolly, M. Easton, S. Lawrie, A. Letchford, J. Pozimski, and P. Savage, Novel integrated design framework for radio frequency quadrupoles, Nucl. Instrum. Methods Phys. Res., Sect. A 735, 240 (2014).
[18] B. Mustapha, A. A. Kolomiets, and P. N. Ostroumov, Full three-dimensional approach to the design and simulation of a radio-frequency quadrupole, Phys. Rev. Accel. Beams 16, 120101 (2013).

[19] CST Studio Suite, http://www.cst.com.

[20] A. A. Kolomiets (private communication).

[21] C. Li, Y. He, and Z. Wang, Optimization design of the RFQ trapezoidal electrode, in Proceedings of the 2015 International Conference on Heavy Ion Accelerator Technology, Yokohama, Japan (JACoW, Yokohama, 2015), p. 203.

[22] The beam dynamics code TRACK, http://www.phy.anl .gov/atlas/TRACK. 